\title{
The Effects of the Combined Use of Platelet-Rich Plasma and Xenograft on Alveolar Socket Healing
}

\author{
Leonardo Isaia ${ }^{1}$, Barbara Isaia ${ }^{1}$, Federica Isaia ${ }^{1}$ and Tonino Traini ${ }^{2 *}$ \\ ${ }^{1}$ Private practice Vigodarzere, Padova, Italy \\ ${ }^{2}$ Department of Medical, Oral and Biotechnological Sciences, University of Chieti-Pescara, Italy
}

Received: February 28, 2018; Published: March 13, 2018

*Corresponding author: Tonino Traini, Department of Medical, Oral and Biotechnological Sciences, University “G d'Annunzio" of Chieti-Pescara, Via dei Vestini, 31; 66100 Chieti, CH, Italy, Tel: 3908713554143; Email: t.traini@unich.it

\section{Abstract}

Objectives: The combination of platelet-rich plasma (PRP) with anorganic bovine bone (ABB) has prospective values for the alveolar bone ridge preservation. The aim of this study was to evaluate the influence of PRP combined with ABB to improve the alveolar socket healing.

Materials and Methods: Ten post-extractions alveolar sockets were filled with ABB particles mixed with PRP gel and covered with a PRP clot membrane. After 4 months of healing, biopsies were collected from the implant beds sites for histomorphometric analysis. Newly formed bone (NFB), marrow spaces (MS), residual graft (RG) bone regenerative index (Br) and structural density index (Ds) were measured and compared to those published in dental literature selected by PICOT method.

Results: In the present study, NFB was $26.5 \%$, MS was $45.6 \%$ and RG was $24.5 \%$ while, using ABB plus saline solution and collagen membrane Vance et al. reported $26 \%$ of NFB, $54 \%$ of MS and $16 \%$ of RG. The difference was not significant ( $p>.05$ ). Conversely, the Br indexes were $2.12 \pm 0.1$ (our study), $1.89 \pm 0.10$ (by Vance) while the Ds indexes were $2.7 \pm 0.11$ (our study), $2.3 \pm 0.12$ 回 (by Vance). The differences were significant $(\mathrm{P}=<.001)$.

Conclusion: The ABB-PRP was useful in alveolar socket preservation, demonstrating a significant increase of bone regenerative index and structural bone density.

Clinical Relevance: The alveolar socket preservation appears to be a more efficient procedure when ABB is mixed to PRP gel and membrane clot is used.

Key words: PRP; Ridge Preservation; Bone Healing, Biomaterials

Abbreviations: NFB: Newly Formed Bone; MS: Marrow Spaces; RG: Residual Graft; BR: Bone Regenerative Index; DS: Structural Density Index; PRP: Platelet-Rich Plasma; TGF: Transforming Growth Factors; PDGF: Platelet Derived Growth Factor; IGF: Insulin-Like Growth Factor; VEGF: Vascular Endothelial Growth Factor; ABB: Anorganic Bovine Bone; BCT: Blood Cell Therapy; GCP: Clinical Practice Guidelines; RKBCT: Regen Kit Blood Cell Therapy; PICOT: Population Intervention Comparison Outcomes and Time

\section{Introduction}

The placement of dental implants in post-extraction sites depends on the amount of alveolar bone present after the bone remodeling process completion. In fact, sometimes after the dental extraction, the alveolar bony walls undergo a heavy resorption, notwithstanding the fact that the bone tissue has regenerative potential. The innate capacity for self-repairing may not be sufficient in extended bone defects; in these cases bone grafting is the treatment of choice. On the other hand, bone grafting is a common and documented procedure that permits the restoration of an adequate bone height for implant placement [1-3]. Several methods of reconstructing or preventing bone defects are available as are types of grafting material [2-4]. Platelet-rich plasma (PRP) first introduced as regenerative material by Ross et al. [5] is a valuable approach with several possible applications in dental and oral surgery for its potential to reduce bleeding and enhance healing processes as well as bone regeneration [6-15]. PRP is obtained from the centrifugation of the patient's autologous blood, and it contains a high concentration of platelets that, when activated, release several growth factors such as the transforming growth factors (TGF-b1 and 2), the platelet-derived growth factor (PDGFBB), insulin-like growth factor-I (IGF-I) and vascular endothelial growth factor (VEGF). 
These are key elements in the promotion of tissue regeneration and osteogenesis [9-10]. Anorganic bovine bone (ABB) (Bio-Oss, Geistlich, Wolhusen, Switzerland) is a xenograft bone substitute biomaterial widely used as a scaffold to allow osteoblasts migration from the neighboring bone and new bone formation [16-17]. Several studies on ABB have demonstrated Histologically and histomorphometrical that new bone formation takes up to 6 months after grafting alveolar ridge defects $[18,19]$. The use of PRP in association with bone graft materials has been proposed in implant surgery in order to enhance and accelerate wound healing and bone regeneration $[11,13,14]$. Promising results have also been obtained in sinus floor elevation and periodontal intrabony defects $[3,12,15]$. Platelets are key factors in hard and soft tissue repair mechanisms. They provide the essential growth factors that are involved in stem cell migration, differentiation inducing at the same time as new extracellular matrix deposition, as well as neovascularization. Blood plasma is essential for cell survival as it contains proteins, nutrients, vitamins, hormones and electrolytes. The plasma-proteins are particularly important molecules of the coagulation process able to form the fibrin clot that will serve as a transitory extracellular matrix for cell migration and proliferation [16-19].

In literature, controversial results were reported regarding the regenerative effects played by the amount of platelet locally adducted [20-23]. It was assessed that concentrations of platelets 1 to 3 times over the baseline show more robust healing rates [20] and at the same time even for platelet concentrations that are too high and may have negative effects inhibiting osteoblasts activity [21], as well as for high growth factor concentrations, which may have inhibitory and cytotoxic effects [22]. The role in the healing of white blood cells (WBC) for subset granulocytes and mononuclear cells is controversial at the same time. Granulocyte neutrophils are the front-line defenders against invading pathogens and are associated with the inflammatory response. They release a large variety of highly active antimicrobial substances and proteases. Uncontrolled release of these factors can cause severe damage to the tissue, delay healing rates and increase the risk of scarring [23]. The lymphocyte and monocytes cells instead have been shown to support the healing process [24-27]. The Regen ${ }^{\mathrm{TM}}$ Blood Cell Therapy (ВСT) tube prepares 4 to $5 \mathrm{ml}$ of Antilogous platelet-rich plasma with a platelet recovery superior to $80 \%$ and a concentration factor 1.6fold. Thanks to the Regen Kit BCT technology, more than $96 \%$ of the granulocytes are removed from Antilogous - Platelet Rich Plasma (A-PRP®).

The few white blood cells still present in A-PRP® are mostly lymphocytes and monocytes. In summary, the BCT technology provides an A-PRP with an $80 \%$ more concentrated platelet with white blood cells mainly of lymphocyte and monocytes types because the granulocytes are removed. The purposes of the present study were to evaluate the in vivo potential of A-PRP mixed with $\mathrm{ABB}$ xenograft to improve the healing of the alveolar sockets preserving the crestal bony walls after teeth extractions and to evaluate histomorphometrically the healing process measuring the bone regenerative index and the bone density structure index based on the proportion rate Histologically determined. The data retrieved in the present study will be compared with those from dental literature for both the same healing time and xenograft type with the exception of PRP use. A null hypothesis of no differences was considered.

\section{Materials and Methods}

\section{Study Design}

The present study is case series clinical trial conducted in one clinical center in accordance with the Good Clinical Practice Guidelines (GCPs) following the recommendations of the Declaration of Helsinki as revised in Fortaleza (2013) for investigations with human subjects. All patients were informed about the benefits and possible risks of the PRP procedure as well as its alternatives. Finally, a signed written consent was obtained. Patientrecruitment, treatment and follow-up visitswereconducted in one private center by twooperators (LI and BI).

\section{Study Population}

Ten patients, six females and four males, with a mean age $( \pm S D)$ of $49.3 \pm 15$ years (Tab. 1 ) who needed tooth avulsion were selected.

General exclusioncriteriawere the following:

a. General contraindications to oralsurgery

b. Patientsimmunosuppressedor irradiated in the head and neck area

c. Uncontrolleddiabetes (HBA1c > 7.5\%)

d. Pregnant or breastfeeding

e. Alcohol or drugsabusers

f. Heavy smokers ( $>20$ cigarettes/day)

g. Psychiatricproblems or unrealisticexpectations

h. Treated or under treatment with intravenous aminobisphosphonates

i. Allergic to bovine collagen

j. $\quad$ Patientsunder treatment of NSAIDs

Local exclusioncriteriaconsisted of the following:

a. Acuteoralinfections

b. Poororalhygiene and motivation (FMPS >30)

c. Uncontrolledperiodontaldisease.

After teeth extraction, the alveolar sockets were filled with ABB biomaterial mixed with PRP gel and subsequently covered with a PRP clot membrane. Patients were also invited not to use NSADs during the 5 days before the surgery so as not to interfere with platelet activity.

\section{A-PRP® Preparation}

The Regen Kit blood cell therapy (RKBCT) product family is indicated by the US FDA for the safe and rapid preparation of PRP. 
The RKBCT uses a physical barrier (thyrotrophic gel) to separate the red blood cells from the plasma and the cellular sediment and sodium citrate as an anticoagulant. The preparation is safe and consistent with a few steps required to prepare 4 to $5 \mathrm{ml}$ of A-PRP® from only $8 \mathrm{ml}$ of blood. The PRP preparation was performed by a single operator (BI) using a dedicated CE mark system, (Regen Kit, Regen Lab, Le Mont sur Lausanne, Switzerland).10 ml of the venous blood was drawn from each patient, placed in the processing tube with the separator gel and centrifuged. After centrifugation, blood was separated; red blood cells were held under the gel while platelets were sedimented on the upper surface of the gel. Platelets were put back in suspension in the plasma by gentle repeated inversions of the tube. Liquid A-PRP® ready to use was in this way obtained and transformed in gel or a clot/membrane through additional steps using other Regenlab systems.

\section{Treatment Procedures}

After performing local anaesthesia by using articaine hydrochloride $4 \%$ with epinephrine 1:100.000 (Septocaine $\AA$,
Septodont, and Cambridge, ON, Canada), the surgical procedures for tooth extractions were performed by an experienced operator (LI). The alveolar sockets were filled with a mixture of plasma A-PRP®gel and particles of ABBin20\% /80\%ratio. Furthermore, A-PRP®clot/ membrane was used to cover the grafted area (Figure 1). After intervention, the patients were prescribed antibiotics for 5 days, amoxicillin $1 \mathrm{~g}$ twice a day or, in allergic patients, clarithromycin $250 \mathrm{mg}$ twice a day. Post-surgical follow-ups were scheduled after 10 days and at monthly intervals to check the course of healing. As postoperative instruction, patients were invited to rinse mouth with $0.12 \%$ Chlorhexidine for 15 days. After four months, a fullthickness flap was raised and a trephine bur (3.5mm diameter) was used instead of the pilot bur to prepare the implant bed (Figure 2). The bone-core biopsies were collected from the grafted areas and then dental implants were inserted in the harvesting sites. For each one of the cases, an intraoral X-Ray was collected to best control the direction of the trephine bur during the retrieval procedure. The $\mathrm{x}$-ray also provided indications on the type of bone present around the biopsy area.
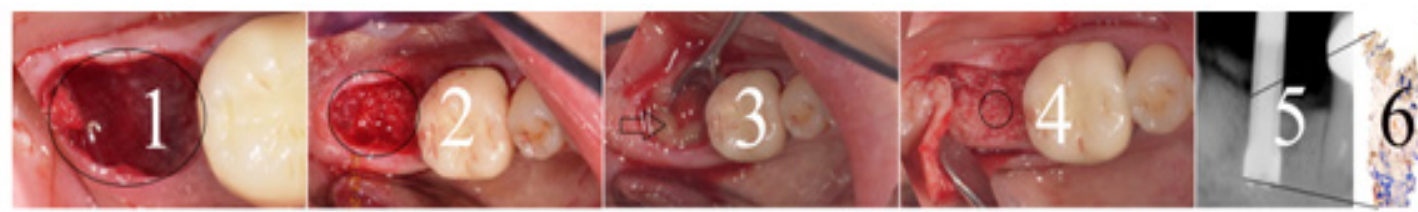

4 Months

Figure 1: Clinical phases of the study.

a. Alveolar socket after tooth extraction.

b. Extraction socket filled with a mixture of plasma A-PRP® gel and ABB particles in a rule of $80 \% / 20 \%$.

c. Application of an A-PRP® clot/membrane.

d. Alveolar socket after 4 months of healing.

e. Rx of trephine bur during the implant bed preparation.

f. Longitudinal section of the bone core (fibrin stain of Ladewig x 12).

g. Implantafterinsertion.

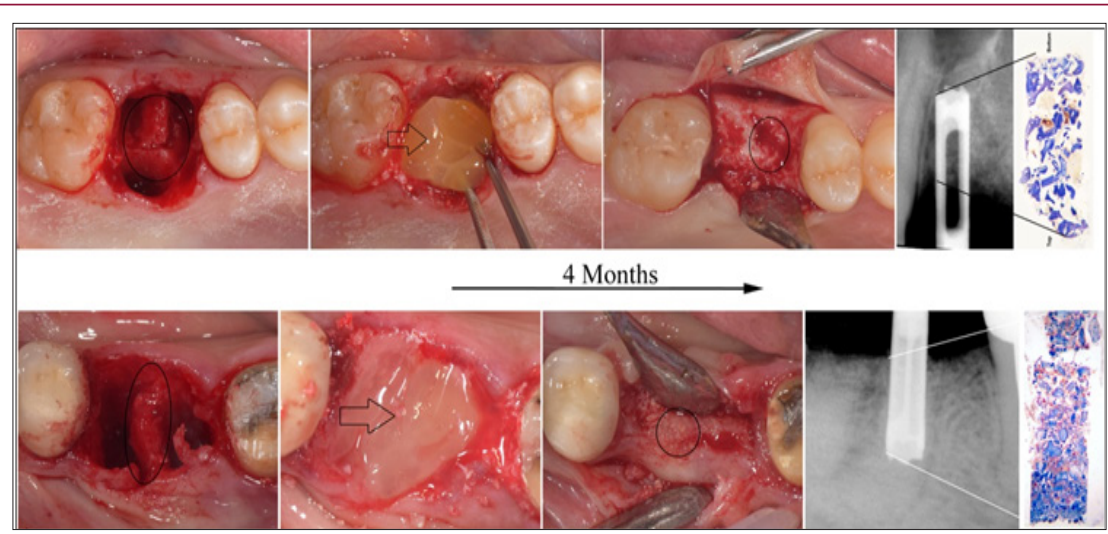

Figure 2: Results of the study. The extraction sockets were always filled with a mixture of ABB and A-PRP® gel and subsequently closed with an A-PRP® clot/membrane (arrows). After 4 months healing, the alveolar sockets appear filled by newly formed bone. The circles indicate the sites where the implant beds were prepared and the bone cores retrieved. The Rx of trephine burs during the implant bed preparation enables us to control the position and depth of the specimens, which were related to the longitudinal section of the bone core for histomorphometric evaluation (fibrin stain of Ladewig $\times 12$ ). 
Table 1: Patient data.

\begin{tabular}{|c|c|c|c|}
\hline I Patients & Age & site & I Gender \\
\hline SE & 55 & 4. & Female \\
\hline RE & 50 & 2. & Female \\
\hline PA & 53 & 2. & Male \\
\hline MF & 34 & 2. & Male \\
\hline EC & 57 & 2. & Female \\
\hline DG & 51 & 5. & Male \\
\hline CM & 22 & 3. & Male \\
\hline GA & 79 & 2. & Female \\
\hline BA & 43 & 5. & Female \\
\hline BM & 49 & 3. & Female \\
\hline \multicolumn{4}{|c|}{ I Mean age (+SD) 49.315} \\
\hline
\end{tabular}

\section{Histological Analysis}

The bone cores, left inside the trephine burs to maintain the orientation, were carefully rinsed with a cold $5 \%$ glucose solution to remove blood while maintaining the correct osmolarity $(278 \mathrm{mOsm} / \mathrm{L})$. The specimens were fixed in $10 \%$ formalin solution at $\mathrm{pH} 7.2$ for a week and dehydrated with a graded series of alcohols. After pre-infiltration treatment in a 50\% resin/alcohol solution (Technovit 7200 VLC, Kulzer, Germany) for ten days, the bone cores were easily removed from the trephine burs with a custom-made plunger and completely embedded in 100\% resin until the specimens became transparent. Finally, the bone cores were oriented and polymerized. A high-precision cutting system (TT System, TMA2, Grottammare, Italy) with a circular diamond disc was used to prepare sections of the specimens along the longitudinal axis of about 50 microns in thickness. The sections were subsequently ground to about $30 \pm 10$ microns under running water with a series of polishing discs, followed by a final polish with 0.1 micron of alumina particles in a micro grinding system (TT System, TMA2, and Grottammare, Italy).

The prepared sections were stained with fibrin stain of Ladewig. The investigation was conducted in a transmitted bright field/ circularly polarized light microscope (BX 51, Olympus America, USA) connected to a high-resolution digital camera (Fine Pix S2 Pro, Fuji Photo Film, Japan). The histomorphometric analysis was performed by means of a software package with image capturing capabilities (Image-Pro plus 6.0, Media Cybernetics Inc, Bethesda, MD, USA) by a trained and experienced operator (TT). To ensure accuracy, the software was calibrated for each experimental image using 'Calibration Wizard', a feature that reports the number of pixels between two selected points of a micrometer scale. The linear remapping of the pixel numbers in microns was used to calibrate the distance.

\section{Regenerative and Structural Density Indexes}

Both the bone regenerative index (Br) and the density structural index (Ds) were calculated following the method introduced and described in depth elsewhere [4]. The indexes were used to best evaluate the histological differences between the present study and that of Vance et al. [28]. The Br indexe mphasizes the relationship among the histomorphometric variables NB, MS and RG percentage. The negative effect off luctuation of every single variable was minimized by weighting. The Ds index was calculated to classify the structural density of the regenerated bone as a function of the treatment. PRP gel and clot membrane vs. saline solution and collagen membrane in alveolar sockets filled with ABB.

\section{Literature Review for Control Group Selection}

The population, intervention, comparison, outcomes and time (PICOT) question used for searching was based on the following: what is the effect of socket filling with a biomaterial after tooth extraction compared to extraction alone in preserving the alveolar bone after a healing time of four months reported in randomized clinical trials in human subjects? The search was limited in the range of years 2000-2017. Additional inclusion criteria for study selection were as follows: bio-oss as xenograft material moistened with sterile saline solution, covered with a collagen barrier membrane, and having a healing time of four months; also, histomorphometric evaluation expressed as rate of new bone, residual graft and marrow spaces have been assessed. Among eleven items founded using the PICOT and time limiting parameters, only one RCT study [28] completely satisfied the inclusion criteria.

\section{Statistical Analysis}

To assess the normal distribution of the values and equality of variances, Shapiro-Wilk and Levene's tests, respectively, were used. Statistical significance of differences between the treatment groups for $\mathrm{Br}$ and Ds variables was done using t-test, while the histomorphometric values expressed by percentage of NFB, MS and RG were analyzed using z-test. Bivariate zero-order Pearson correlation analysis was done to clarify the relationship among the variables bone area of retrieved tissue (BA), the area of newly formed bone (NFB), the area of marrow spaces (MS) and finally the area of residual graft particles (RG). Type I error was set at 0.05 and all analyses were done using SPSS version 18 (SPSS Inc., Chicago, IL, USA).

\section{Results}

\section{Clinical}

From a practical point of view, the use of A-PRP®gel to mix the particles of ABBgreatly facilitates and improves the local application of the mixture compared to saline or blood used conventionally (Figures 1,2).

The application of an A-PRP®clot/membrane reduced the postoperative bleeding. The pain and the postoperative swelling were reduced so much that the patients did not take any pain medications.

\section{Histo Morphometry}

The total area (sum) of repaired tissues obtained with the longitudinal sections was $113 \mathrm{~mm}^{2}$ with a mean area $( \pm$ SD) of each section of $11.3( \pm 3.8) \mathrm{mm}^{2}(95 \% \mathrm{CI}$ of mean 2.76) (Figure 3 \&Table $2)$. After four months of healing, the mean area $( \pm S D)$ of new bone was $3.0( \pm 1.8) \mathrm{mm}^{2}(95 \% \mathrm{CI}$ of mean 1.3$)$, the marrow spaces was $5.15( \pm 2.1) \mathrm{mm}^{2}(95 \% \mathrm{CI}$ of mean 1.5$)$ and finally the biomaterial 
particles was $3.1( \pm 2.5) \mathrm{mm}^{2}(95 \%$ CI of mean 1.8). Pearson correlation analysis (2-Tailed) showed a positive significant correlation between BA and MS ( $r=0.8, n=10, p=.004)$ and MS and NFB $(r=0.6, n=10, p=.048)$. While a positive, but not significant, correlation was present between NBF and BA ( $r=0.5, n=10, p=.08)$.
Differently, negative not significant correlations were noted among $R G$ and NBF ( $r=-0.3, n=10, p=.28) ; R G$ and MS ( $r=-0.4, n=10, p=.90)$. The cumulative percentage of newly formed bone was $26.5 \%$, the marrow spaces $45.6 \%$ and the residual graft particles $27.7 \%$.

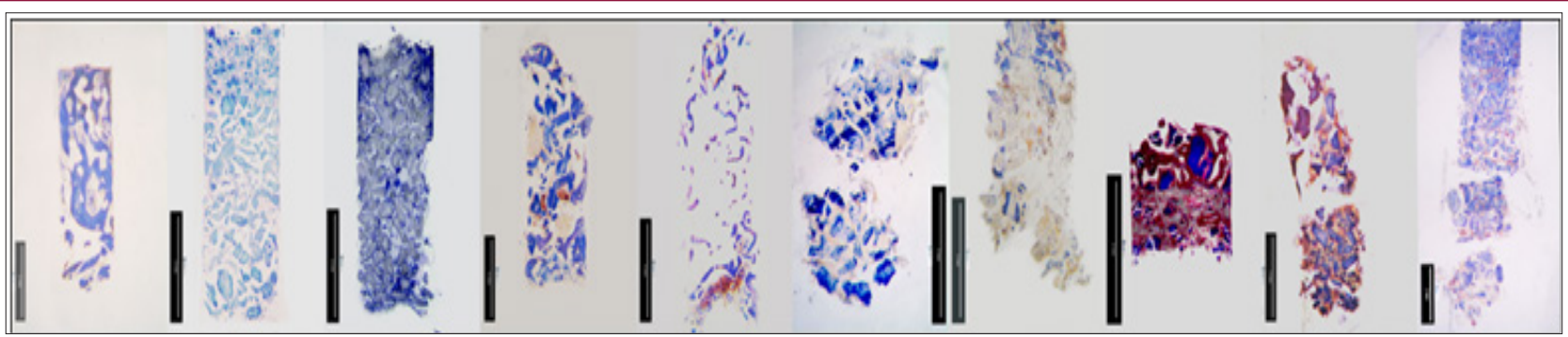

Figure 3: Collection of the bone cores evaluated in the present study. The majority of the bone cores retrieved using the trephine bur conserved the microstructure notwithstanding the mechanical stress produced by the drilling procedure. This observation demonstrates that in four months of healing newly formed bone presents an adequate mechanical resistance. (fibrin stain of Ladewig $\times 12$ ).

Table 2: Summary of historical results.

\begin{tabular}{|c|c|c|c|c|}
\hline Patients & Biopsy area & New bone & $\begin{array}{c}\text { Marrow } \\
\text { space }\end{array}$ & $\begin{array}{c}\text { Biomaterial } \\
\text { particles }\end{array}$ \\
\hline SE & 18.85 & 3.34 & 7.55 & 7.96 \\
\hline RE & 12.88 & 5.16 & 7.61 & 0.11 \\
\hline PA & 11.83 & 3.21 & 4.54 & 4.08 \\
\hline MF & 10.06 & 2.64 & 3.63 & 3.79 \\
\hline EC & 13.43 & 5.21 & 7.89 & 0.33 \\
\hline DG & 7.79 & 0.12 & 3.53 & 4.14 \\
\hline CM & 11.39 & 3.56 & 3.25 & 4.58 \\
\hline GA & 11.08 & 0.78 & 5.79 & 4.51 \\
\hline BA & 11.73 & 4.72 & 5.85 & 1.16 \\
\hline BM & 3.81 & 1.23 & 1.90 & 0.68 \\
\hline Sum & $113 \mathrm{~mm}^{2}$ & $29.97 \mathrm{~mm}^{2}$ & $51.54 \mathrm{~mm}^{2}$ & $31.34 \mathrm{~mm}^{2}$ \\
\hline Area percentage & $265 \%$ & $46 . \%$ & $273 \%$ \\
\hline
\end{tabular}

The control group retrieved by the study of Vance et al. [28] after four months of healing showed $26 \%$ of new bone, $54 \%$ of marrow spaces and $16 \%$ of residual graft particles (Figure 4). No statistically significant differences were found $(\mathrm{P}>.05)$ (Table $3)$. The Br index (Mean \pm SD) in the present study was $2.12 \pm 0.11$ $(\mathrm{N}=10)$ while in the control group (BDX from Vance et al. [28] it was $1.89 \pm \times \mathbb{X}$. The difference was highly statistically significant $(\mathrm{P}=<.001)$. The Ds index $($ Mean $\pm \mathrm{SD})$ for the present study was $2.7 \pm 0.11(\mathrm{~N}=10)$ while for the control group (BDX from Vance et al. [28] it was 2.3 \pm The difference was highly statistically significant $(\mathrm{P}=<.001)$ (Figure 4) and (Table 3 ). The repaired tissue at crestal level consisted of biomaterial particles embedded in a dense connective tissue with few areas of newly formed bone (Figure 5) from a to c. Deep inside the alveolar socket, several areas of newly formed bone were present; these were mainly related to areas with poorly compacted biomaterial particles (Figure $5 \mathrm{~d} \& 5 \mathrm{e}$ ).

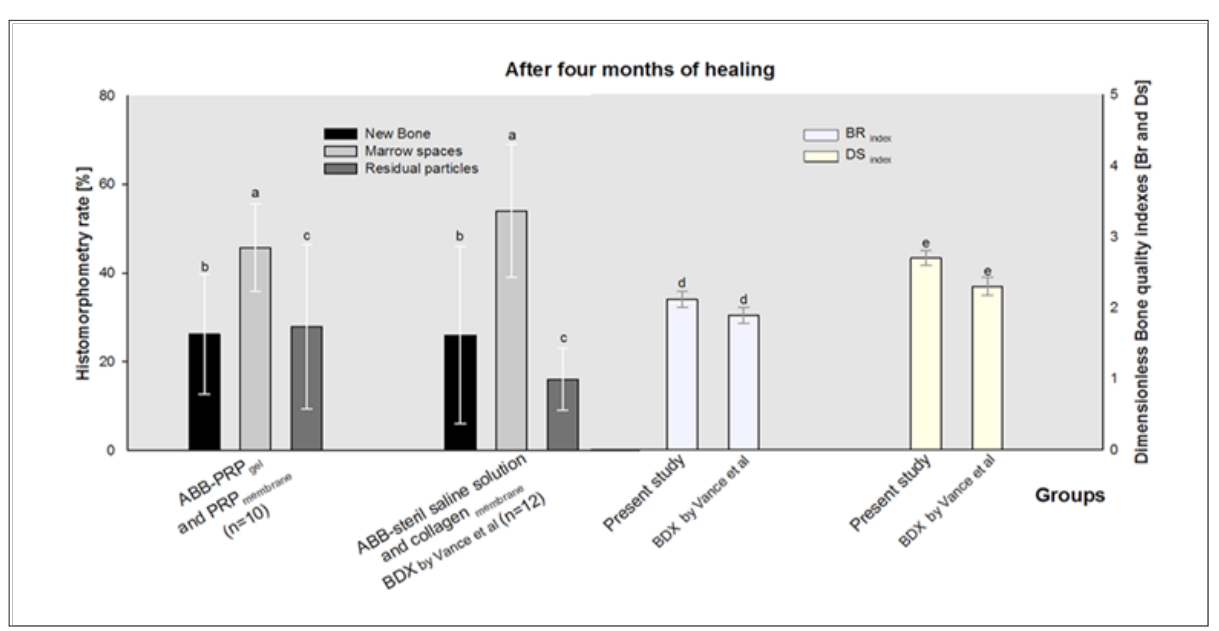

Figure 4: Graph of the results in comparison with Vance et al.28 for histomorphometric and quality of the bone. The Z-test with Yates correction applied to histomorphometric results for new bone, marrow spaces and residual particles showed an insignificant difference in the proportions between $b(p=0.645)$, a $(p=0.975)$ and $c(p=0.885)$, respectively. Conversely, for the bone quality indexes, (BR and DS) the differences were statistically significant $(p<.001)$ with $t$-test for independent samples. 


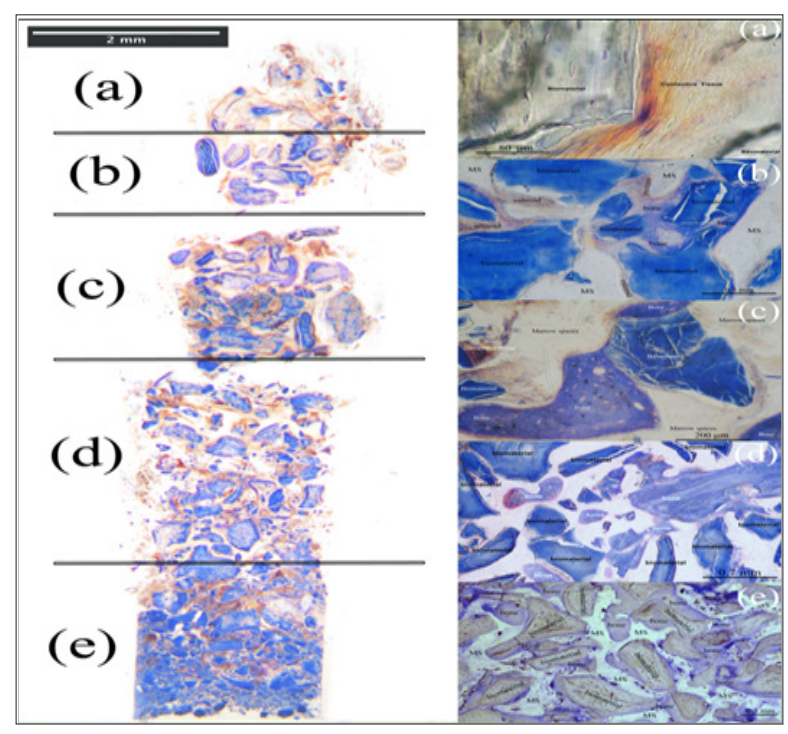

Figure 5: The histological evaluation along the bone core starting from the crestal side

a) Until the deep area of the alveolar socket and e) showed an increasing amount of newly formed bone.

a. At $\times 15$ and at high magnification (x 400), biomaterial particles surrounded by a dense strut of collagen fibres were visible.

b. At x 15 and at high magnification (x 400), an area of newly formed bone always associated with osteoid tissue around the biomaterial particles was seen.

c. At $x 15$ and at high magnification ( $x 400$ ), the area of new bone appeared more represented.

d. At $\times 15$ and $\times 200$ magnifications, the new bone formation was mainly associated with the area where the biomaterial particles appeared to be less compacted.

e. At $\times 15$ and $\times 200$ magnifications, the new bone with biomaterial particles embedded appeared to be disposed in a network of repaired tissue.

Table 3: Sum mary of the statistical data.

\begin{tabular}{|c|c|c|c|c|c|c|c|}
\hline \multicolumn{8}{|c|}{ Z-test Yates of correction applied to calculations } \\
\hline Group name & variables & $\mathbf{n}$ & rate & Diff of rate & SEr & $\mathbf{Z}$ & $\mathbf{P}$ \\
\hline Present study & \multirow{2}{*}{ NFB } & 10 & 26.2 & \multirow{2}{*}{0.002} & \multirow{2}{*}{0.188} & \multirow{2}{*}{0.477} & \multirow{2}{*}{0.633} \\
\hline Vance et al. & & 12 & 26 & & & & \\
\hline \multicolumn{8}{|c|}{$95 \%$ CI for differences:- 0.367 to 0.371} \\
\hline Present study & \multirow{2}{*}{$\mathrm{RG}^{\mathrm{v}}$} & 10 & 45.7 & \multirow{2}{*}{-0.0830} & \multirow{2}{*}{0.214} & \multirow{2}{*}{-0.0405} & \multirow{2}{*}{0.968} \\
\hline Vance et al. & & 12 & 54 & & & & \\
\hline \multicolumn{8}{|c|}{ 95\% CI for differences:- 0.503 to 0.337} \\
\hline \multicolumn{8}{|c|}{ t-test } \\
\hline Group name & variables & $\mathrm{n}$ & mean & SD & SEM & \multicolumn{2}{|c|}{$\mathrm{P}$} \\
\hline Present study & $\mathrm{Br}^{\mathrm{d}}$ & 10 & 2.12 & 0.11 & 0.0351 & \multirow{3}{*}{\multicolumn{2}{|c|}{$<.001$}} \\
\hline Vance et al. & & 12 & 1.89 & 0.10 & 0.0306 & & \\
\hline \multicolumn{2}{|c|}{ Mean differences } & \multicolumn{2}{|c|}{0.230} & & & & \\
\hline \multicolumn{8}{|c|}{$\mathrm{t}=4.961$ with 20 de areas of freedom. $95 \%$ CI for difference of means: 0.133 to 0.327} \\
\hline \multicolumn{8}{|c|}{ Paver of performed test with alpha $=0.050: 0993$} \\
\hline Present study & Dse & 10 & 2.7 & 0.11 & 0.0354 & \multirow{3}{*}{\multicolumn{2}{|c|}{$<.001$}} \\
\hline Vance et al. & & 12 & 2.3 & 0.12 & 0.0364 & & \\
\hline \multicolumn{2}{|c|}{ Mean differences } & \multicolumn{2}{|c|}{0.400} & & & & \\
\hline \multicolumn{8}{|c|}{$\mathrm{t}=7.791$ with 20 de trees of "reedom. $95 \%$ CI for difference of means: 0.293 to 0.507} \\
\hline \multicolumn{8}{|c|}{ power of performed test with alpha $=0.050: 1.000$} \\
\hline
\end{tabular}




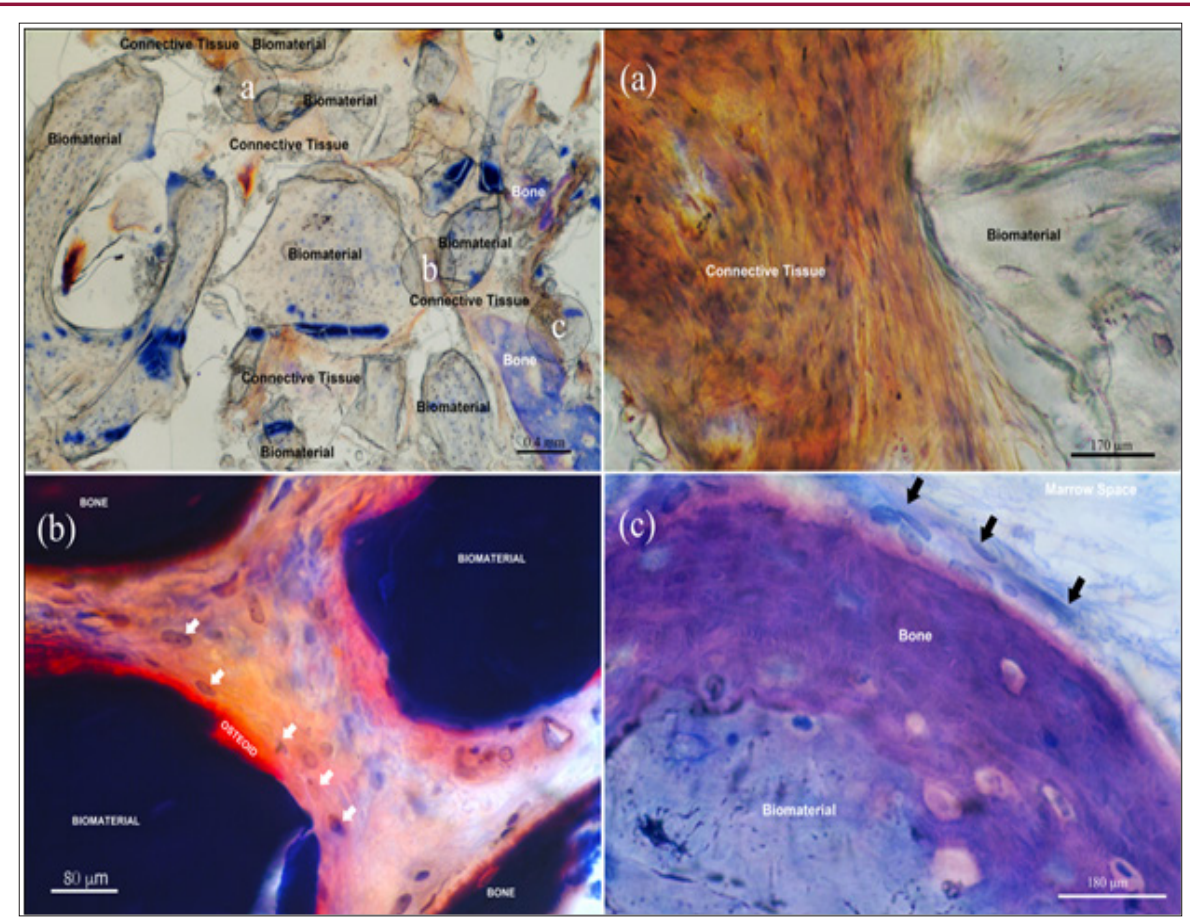

Figure 6: Image in the upper left corner at x 20 magnification of a bone core. The labelled areas (a), (b) and (c) at x 1000 magnifications.

a. the crestal side of the core shows an ABB biomaterial particle surrounded by dense connective tissue fibres.

b. $\quad 0.5 \mathrm{~mm}$ deeply the ABB particles appear surrounded by the osteoid and rim of osteoblastic cells (white arrows).

c. At $1.8 \mathrm{~mm}$ deeply respect to the point (a) the ABB particles appear to be surrounded by new bone with several entrapped plump osteocytes and osteoblastic cells (black arrows).

\section{Discussion}

The healing of the extraction socket is a cascade of events, and it occurs through a number of processes, including bleeding, clotting, and formation of the provisional matrix. The replacement of this matrix starts with re-epithelialization and connective tissue growth in the superficial area of the alveolar socket, while deep inside the socket, the transitional matrix maturation starts with mineralized bone nodules laid-down by static cords of osteoblasts (static osteogenesis) and runs with woven and lamellar bone formed by movable osteoblastic laminae [29]. After six weeks, bone trabeculae appeared to be in an advanced phase of mineralization; the thickening process of the trabeculae is associated with movable osteoblastic laminae (dynamic osteogenesis). The bone deposition that will be completed in 4-6 months [29-31]. The main consequence of tooth extraction is a significant resorption of the bony walls of the alveolar socket. Pietrokovski \& Massler [32] and Schropp et al. [33] have shown that the edentulous site remodels in all dimensions involving the soft tissues of the extraction site. The adaptive changes clinically may appear as deformations of the jaw.

Ultimately, the indications for alveolar socket preservation include maintenance of the existing hard and soft tissues to simplify subsequent treatments such as animplant or denture placement. A variety of materials are available for post-extraction ridge preservation. Nevertheless, to achieve optimal results, all grafts require adequate blood supply, mechanical support, and osteogenic cells supplied by the host [34]. It was reported by Kotsakis et al. [35] anhigher alveolar bone preservation of human sockets if filled with blood clot and anorganic bovine bone, when compared to blood clot and bioactive glass ceramics. The employment of platelet-rich plasma (PRP) constitutes a relatively innovative technique to improve bone healing. Some studies have been conducted to verify the effectiveness of PRP also in combination with different bone substitutes. These studies have found that PRP combined with autogenous bone provides considerably faster bone regeneration results, radiographic and histomorphometrically, besides indicating denser bone [35].Variable successful results have been demonstrated when the PRP is added to an allograft [36]. Alissa et al. [37], in a pilot study on the effect of PRP on the healing of extraction sockets, reported a significantly improved soft tissue healing in patients treated with PRP compared with those of the control group (no treatment).

Likewise, recently, Celio-Mariano et al. [38] in a study of mandibular third molars after extraction showed a greater radiographic bone density in the PRP group compared to the control group, thereby demonstrating a significant improvement in bone healing in the sockets. Yilmaz et al. [20] investigated the effectiveness of PRP and a bovine-derived xenograft (BDX) combination on early wound healing in deep intrabony defects. One year after surgery, the results showed that PRP in combination with BDX leads to a significantly favorable clinical improvement in deep 
intrabony periodontal defects. In the present investigation, the null hypothesis under test was rejected. Our findings are in accordance with the previous studies. In fact, for the PRP group, the bone regenerative index, as well as the bone density, was significantly higher $(\mathrm{p}<.001)$.About the absence of statistical significance for the histomorphometric results, we believe that the variables expressed as percentage and used to compare groups with different treatments hides the slight differences due to the absence of normalization. Particularly with respect to the mean's standard deviations of the areas calculated in percentage to the total area of the biopsy.

In other words, the use of bone histomorphometry to evaluate the effects of regenerative techniques in the presence of unresorbable biomaterials is less sensitive, since it is more difficult to detect a statistically significant difference with the sample number that could be handled in a clinical study. To strengthen this consideration, there are the results of the bivariate zero-order Pearson correlation analysis (2-Tailed) on the relationship among the histomorphometric variables: BA, NFB, MS and RG. Recalling the results, when the dimension of the bone core (BA) increases, the extension of the marrow spaces significantly increases; at the same time, the marrow space increase results in a significant increase of the amount of newly formed bone. This last aspect indicates the formation of the new bone needs of a certain amount of medullary spaces. In sites where compacted particles of ABB were present, a reduced (negative correlation with $r=-0.45$ ) of bone marrow space was present, and there was less amount of newly formed bone (negative correlation with $\mathrm{r}=-0.37$ ).

This last observation demonstrates that it is better not to compress too much the biomaterial particles during the surgical procedure, and it is advisable not to use a single biomaterial for bone regeneration but instead use a compound of several (almost two) materials at different speeds of resorption. A positive but not significant correlation was noted between RG and BA, underlining an increase of particles also as a consequence of the size of the biopsy. It is our opinion that the ABB material, thanks to a very slow resorption rate, assures a mechanical support during the healing process. While the autogenous PRP gel, in addition to stabilizing and maintaining the graft in position during the surgical procedure, contributes locally to enhance the regional acceleratory phenomenon (RAP).In fact, the healing starts with the blood clot formation, due to the platelet cytokines release(PDGF, TGF- $\beta$, IGF1 ) and receives a boost from cytokines concentrated in the PRP gel.

At the same time, leukocytes and monocytes regulate the inflammatory phenomena associated with grafting. Immune responses are crucial not only for host defense against pathogens but also for tissue repair after injury. Lymphocytes are involved in the healing process after tissue injury, including bone fracture and muscle damage [27]. Moreover, the presence of a transitory matrix around the ABB particles facilitates the cellular migration throughout the fibrin network into the regenerative site as well as the development of neo-angiogenesis and vascularization, promoting the healing of the site. The autogenously membrane clot placed over the biomaterial facilitates the cellular migration improving the soft tissue healing and maturation. Further investigations are needed to evaluate the in-depth interaction between PRP and the xenograft and the best rate of proportion between the two components.

\section{Conclusion}

The combination of xenograft particles of ABB with autogenous PRP gel enriched with lymphocytes and monocytes and PRP membrane provides, at the same time, a significant increase of the bone regenerative index and of the bone density index, favoring the crestal bone preservation. After four months of healing, all the treated sites appeared with enough bone. The membrane of autogenous fibrin-clot, positioned to cover the alveolus, was associated with a reduced postoperative pain. Clinically, there have been better and more predictable results in terms of preservation of alveolar bone.

\section{Acknowledgment}

Experimental materials were provided by RegenLab, Le Mont sur Lausanne, Switzerland. FI collected the clinical data and revised the paper.

\section{References}

1. Aghaloo TL, Moy PK (2007) which hard tissue augmentation techniques are the most successful in furnishing bony support for implant placement. The International Journal of Oral \& Maxillofacial Implants 22: $49-70$

2. Al Nawas B, Schiegnitz E (2014) Augmentation procedures using bone substitute materials or autogenous bone-a systematic review and metaanalysis. EuropeanJournal of OralImplantology 7(2): 219-234.

3. Esposito M, Grusovin MG, Coulthard P, Worthington HV (2006) The efficacy of various bone augmentation procedures for dental implants: A Cochrane systematic review of randomized controlled clinical trials. The International Journal of Oral \& Maxillofacial Implants 21(5): 696-710.

4. Traini T, Piattelli A, Caputi S, Degidi M, Mangano C, et al. (2015) Regeneration of human bone using different bone substitute biomaterials. Clinical Implant Dentistry and Related Research 17(1): 150-162.

5. Ross R, Glomset J, Kariya B, Harker L (1974) A platelet-dependent serum factor that stimulates the proliferation of arterial smooth muscle cells in vitro. Proceedings of the National Academy of Sciences of the United States of America 71(4): 1207-1210.

6. Antonino Albanese, Maria E Licata, Bianca Polizzi, Giuseppina Campisi (2013) Platelet-rich plasma (PRP) in dental and oral surgery: From the wound healing to bone regeneration. Immunity \& Ageing 10(1): 23.

7. Forni F, Marzagalli M, Tesei P, Grassi A (2013) Platelet gel: Applications in dental regenerative surgery. Blood Transfusion 11(1): 102-107.

8. Kumar KA, Rao JB, Pavan Kumar B, Mohan AP, Patil K, et al. (2013) A prospective study involving the use of platelet-rich plasma in enhancing the uptake of bone grafts in the oral and maxillofacial region. Journal of Maxillofacial Oral Surgery 12(4): 387-394.

9. Nikolidakis D, Jansen JA (2008) The biology of platelet-rich plasma and itsapplication in oral surgery: Literature review. TissueEngineering: Part B 14(3): 249-258.

10. Anitua E, Andia I, Ardanza B, Nurden P, NurdenAT (2004) Autologous platelets as source of proteins for healing and tissue regeneration. Thrombosis Haemostasis 91(1): 4-15.

11. Del Fabbro M, Bortolin M, Taschieri S, Weinstein R (2011) Is platelet concentrate advantageous for the surgical treatment of periodontal 
diseases? A systematicreview and meta-analysis. Journal of Periodontology 82(8): 1100-1111.

12. Khairy NM, Shendy EE, Askar NA, El Rouby DH (2013) Effect of plateletrich plasma on bone regeneration in maxillary sinus augmentation (randomized clinical trial). International Journal of Oral Maxillofacial Surgery 42(2): 249-255.

13. Inchingolo F, Tatullo M, Marrelli M, Inchingolo AM, Scacco S, et al. (2010) Trial with Platelet-Rich Fibrin and Bio-Oss used as grafting materials in the treatment of the severe maxillar bone atrophy: Clinical and radiological evaluations. EuropeanReview for Medical and PharmacologicalSciences 14(12): 1075-1084.

14. Poeschl PW, Ziya Ghazvini F, Schicho K, Buchta C, Moser D, et al. (2012) Application of platelet-rich plasma for enhancedbone regeneration in grafted sinus. Journal of Oral and Maxillofacial Surgery 70(3): 657-664.

15. Hanna R, Trejo PM, Weltman RL (2004) Treatment of intrabony defects with bovine-derived xenograft alone and in combination with plateletrich plasma: A randomized clinical trial. Journal of Periodontology 75(12): 1668-1677.

16. Tadjoedin ES, de Lange GL, Bronckers AL, Lyaruu DM, Burger EH (2003) Deproteinizedcancellous bovine bone (Bio-Oss) as bone substitute for sinus floor elevation. A retrospective, histomorphometrical study of five cases. Journal of Clinical Periodontology 30(3): 261-270.

17. TrubianiO, Fulle S, Traini T, Paludi M, la Rovere R, et al. (2010) Functional assay, expression of growth factors and proteins modulating bonearrangement in human osteoblasts seeded on an anorganic bovine bone biomaterial. European Cell \& Materials 20: 72-83.

18. Zitzmann N, Schärer P, Marinello C, Schüpbach P, Berglundh T (2001) Alveolar ridge augmentation with Bio-Oss $\AA$ : A histologic study in humans. The International Journal of Periodontics \& Restorative Dentistry 21(3): 288-295.

19. Orsini G, Traini T, Scarano A, Degidi M, Perrotti V, et al. (2005) Maxillary sinus augmentation with Bio-Oss particles: A light, scanning, and transmission electron microscopy study in man. Journal of Biomedical Material Research Part B: Applied Biomaterials 74(1): 448-457.

20. Rappl LM (2011) Effect of platelet-rich plasma gel in a physiologically relevant platelet concentration on wounds in persons with spinal cord injury. International Wound Journal 8(2): 187-195.

21. Weibrich G, Hansen T, Kleis W, Buch R, Hitzler WE (2004) Effect of platelet concentration in platelet-rich plasma on peri-implant bone regeneration. Bone 34(4): 665-671.

22. Graziani F, Ivanovski S, Cei S, Ducci F, Tonetti M, et al. (2006) The in vitro effect of different PRP concentrations on osteoblasts and fibroblasts. Clinical Oral Implants Research 17(2): 212-219.

23. Brubaker AL, Schneider DF, Kovacs EJ (2011) Neutrophils and natural killer T cells as negative regulators of wound healing. Expert Review of Dermatology 6(1): 5-8.

24. Barbul A, Shawe T, Rotter SM, Efron JE, Wasserkrug HL, et al. (1989) Wound healing in nude mice: A study on the regulatory role of lymphocytes in fibroplasia. Surgery 105(6): 764-769.

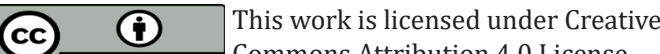

Submission Link: https://biomedres.us/submit-manuscript.php
25. Brancato SK, Albina JE (2011) Wound macrophages as key regulators of repair: Origin, phenotype, and function of ion. American Journal Pathology 178(1): 19-25.

26. Hsieh JY, Smith TD, Meli VS, Tran TN, Botvinick EL, et al. (2017) Differential regulation of macrophage inflammatory activation by fibrin and fibrinogen. Acta Biomaterialia 47: 14-24.

27. Ono T, Okamoto K, Nakashima T, Nitta T, Hori S, et al. (2016) IL-17producing $\gamma \delta \mathrm{T}$ cellsenhance bone regeneration. Nature Communications 7: 109-128.

28. Vance GS, Greenwell H, Miller RL, Hill M, Johnston H, et al. (2004) Comparison of an allograft in an experimental putty carrier and a bovine-derived xenograft used in ridgepreservation: A clinical and histologicstudy in humans. Int J Oral Maxillofac Implants 19(4): 491497.

29. Traini $\mathrm{T}$ (2013) The development of the alveolar bone by static osteogenesis: Microanatomy and clinical implications. European Journal of Surgery 4(2): 29-37.

30. Pagni G, Pellegrini G, Giannobile WV, Rasperini G (2012) Postextraction alveolar ridge preservation: Biological basis and treatments. International Journal of Dentistry.

31. Barone A, Ricci M, Tonelli P, Santini S, Covani U (2013) Tissue changes of extraction sockets in humans: A comparison of spontaneous healing vs. ridge preservation with secondary soft tissue healing. Clinical OralImplantsResearch 24(11): 1231-1237.

32. Pietrokovski J, Massler M (1967) Ridge remodeling after tooth extraction in rats. Journal of Dental Research 46(1): 222-231.

33. Schropp L, Wenzel A, Kostopoulos L, Karring T (2003) Bone healing and soft tissue contour changes following single-tooth extraction: A clinical and radiographic 12-month prospective study. The International Journal of Periodontics \& Restorative Dentistry 23(4): 313-323.

34. Darby I (2011) Periodontal materials. Australian Dental Journal 56(1): 107-118.

35. Tarantino R, Donnarumma P, Mancarella C, Rullo M, Ferrazza G, et al. (2014) Posterolateral arthrodesis in lumbar spine surgery using autologous platelet-rich plasma and cancellous bone substitute: An osteoinductive and osteoconductive effect. Global Spine J 4(3): 137-142.

36. Kotsakis GA, Salama M, Chrepa V, Hinrichs JE, Gaillard P (2014) A randomized, blinded, controlled clinical study of particulateanorganic bovine bone mineral and calciumphosphosilicate putty bone substitutes for socketpreservation. Int J Oral Maxillo facImplants 29(1): 141-151.

37. Alissa R, Esposito M, Horner K, Oliver R (2010) The influence of platelet-rich plasma on the healing of extraction sockets: An explorative randomized clinical trial. European Journal of OralImplantology 3(2): 121-134.

38. Célio Mariano R, de Melo WM, Carneiro Avelino C (2012) Comparative radiographic evaluation of alveolar bone healing associated with autologous platelet-rich plasma after impacted mandibular third molar surgery. Journal of Oral and Maxillo facial Surgery 70(1): 19-24.

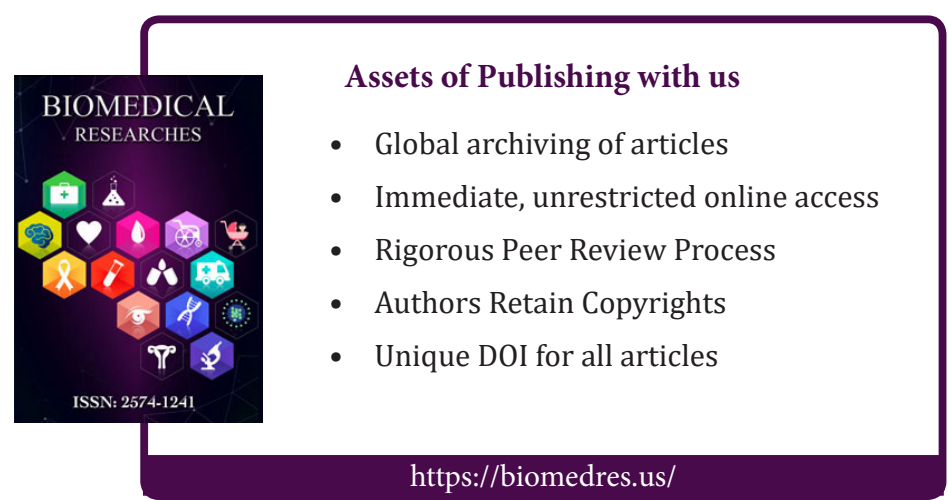

